Pacific Journal of Mathematics

FIXED POINT SETS OF HOMOTOPIES 


\title{
FIXED POINT SETS OF HOMOTOPIES
}

\author{
Helga SCHIRMER
}

\begin{abstract}
In recent years it has been shown that many spaces have the so-called complete invariance property, i.e. that every closed and nonempty subset of them can be realized as the fixed point set of a continuous selfmap. Here a related result is obtained for homotopies $H$ : $X \times I \rightarrow X$ rather than selfmaps of a space $X$. The theorem proved here states that if $P$ is a compact and connected polyhedron without local cut points and $K \subset P \times I$ a closed set which contains a continuum intersecting both $X \times 0$ and $X \times 1$, then there exists a homotopy $H: P \times I \rightarrow P$ with fixed point set $K$.
\end{abstract}

1. The result. In recent years several authors have been interested in the realization of a subset $A$ of a topological space $X$ as the fixed point set Fix $f=\{x \in X \mid f(x)=x\}$ of a continuous selfmap of $X$ under minimal assumptions on $A$. If $X$ is Hausdorff, then $A$ is necessarily closed, and in order to avoid questions concerning the fixed point property it has also been assumed that $A$ is non-empty. In many cases these assumptions suffice. $X$ is called a space with the complete invariance property (CIP) if every closed and non-empty subset $A$ of $X$ is the fixed point set of a map $f: X \rightarrow X$, and it is known that the class of spaces with the CIP includes all convex subsets of a normed linear space, compact topological manifolds, locally finite simplicial complexes with the weak topology, locally compact metrizable topological groups and 1-dimensional Peano continua. A survey of these results, which are due to Boju Jiang (Po-Chu Chiang), J. R. Martin, S. B. Nadler, Jr., H. Robbins, H. Schirmer, E. D. Tymchatyn and L. E. Ward, Jr., and references can be found in [8].

In this paper a similar problem is considered for homotopies $H$ : $X \times I \rightarrow X$, where $I=[0,1]$ is the unit interval. The fixed point set of a homotopy $H$ is defined by

$$
\text { Fix } H=\{(x, t) \in X \times I \mid H(x, t)=x\},
$$

and hence we are interested in minimal assumptions on a set $K \subset X \times I$ under which $K$ can be realized as the fixed point set of a homotopy of $X$. Again $K$ is closed if $X$ is Hausdorff, and we shall also assume that $K \cap(X \times t) \neq \varnothing$ for every $t \in I$. But in general this is not enough, as the continuity of $H$ implies conditions on the change of $K \cap(X \times t)$ with 
varying $t$. To clarify the situation, we say that a set $A \subset X \times 0$ is joined to $B \subset X \times 1$ by a continuum (i.e. a compact and connected space) $C \subset X \times I$ if $C \cap(X \times 0) \subset A$ and $C \cap(X \times 1) \subset B$, and use ind $(f, X)$ to denote the fixed point index of the map $f: X \rightarrow X$. Then the following proposition is an easy consequence of a rather complex theorem by F. Browder (see [1], Theorem 1).

Proposition. Let $X$ be a compact ANR and $H: X \times I \rightarrow X$ a homotopy from $f=H \mid X \times 0$ to $g=H \mid X \times 1$. If ind $(f, X) \neq 0$, then there exists a continuum $C$ in Fix $H$ joining Fix $f$ and Fix $g$.

(Browder's theorem only implies the existence of a connected set $C_{1}$ in Fix $H$ joining Fix $f$ and Fix $g$. To obtain a continuum, simply take the closure of $C_{1}$.)

The proposition shows that in order to realize the closed set $K \subset X \times I$ as the fixed point set of a homotopy it is generally necessary to assume that $K$ contains a continuum joining $X \times 0$ and $X \times 1$.

We show that these assumptions on $K$ are sufficient for certain polyhedra. Recall that a local cut point (or separating point) is a point $x$ which has a connected neighbourhood $N$ so that $N-x$ is not connected. The purpose of this paper is the proof of the following result.

Theorem. Let $P$ be a compact and connected polyhedron without local cut points, and $K \subset P \times I$ a closed set which contains a continuum $C$ joining $P \times 0$ and $P \times 1$. Then there exists a homotopy $H: P \times I \rightarrow P$ with Fix $H=K$.

The assumptions on $P$ in the Theorem are e.g. satisfied by those polyhedra for which it is presently known that the Nielsen number $N(f)$ of a selfmap $f$ can be realized by a map homotopic to $f$ (see [3], Main Theorem; compare also [2], Ch. VIII D, Theorem 1, and [9], Theorem 2.4) and by all compact triangulable manifolds of dimension $\geq 2$. It is easy to check directly that the Theorem also holds for all 1-dimensional manifolds. Nevertheless the class of spaces for which we prove the Theorem is more restrictive than the class of spaces which are known to have the CIP, even if attention is confined to polyhedra. The reason is that we consider only the case where $H$ can be obtained via path fields, and hence $H \mid P \times 0$ is homotopic to the identity map of $P$. The path field method of proof establishes the CIP for 2-dimensionally connected polyhedra [8], i.e. for polyhera where every maximal simplex is of dimension $\geq 2$ and where 
every two maximal simplexes $\tau, \tau^{\prime} \in P$ are connected by a chain of maximal simplexes $\tau_{1}=\tau, \tau_{2}, \ldots, \tau_{r}=\tau^{\prime}$ so that $\bar{\tau}_{i} \cap \bar{\tau}_{i+1}$ is of dimension at least one for $i=1,2, \ldots, r-1$. (See $\$ 2$ for the notation used.) A polyhedron without local cut points is 2-dimensionally connected, but the converse is not true. The condition that $P$ has no local cut points can be considered as a localization of 2-dimensional connectedness, and is needed in the proof of the Theorem to ensure that certain subpolyhedra are 2-dimensionally connected.

There is little doubt that the Theorem holds for a much wider class of spaces. Combining the techniques of this paper with those of [4] will likely suffice to establish it for all locally finite simplicial complexes with the weak topology. But while such a proof is very feasible, its length may prevent it from being enjoyable.

2. Background: Proximity maps and path fields. We write $(P, T)$ for a polyhedron $P$ with triangulation $T$. An open simplex of $(P, T)$ is denoted by $\tau$, the corresponding closed simplex by $\bar{\tau}$, and its boundary by $\dot{\tau}$. Let $\kappa(x)$ stand for the carrier of the point $x \in P$. The sets

$$
\text { st } \tau=\bigcup\{y \in P \mid \tau \in \bar{\kappa}(y)\}
$$

and

$$
V(x)=\{y \in P \mid \bar{\kappa}(x) \cap \bar{\kappa}(y) \neq \varnothing\}
$$

are open neighbourhoods of $\tau$ resp. $x$. Hence if $X$ is a subspace of $P$, then

$$
V(X)=\bigcup\{V(x) \mid x \in X\}
$$

is an open neighbourhood of $X$. Note that $V(\kappa(x))=V(x)$, and that $V(\tau) \subset V(\sigma)$ if the simplex $\tau$ is a face of the simplex $\sigma$. A map $f: X \rightarrow P$ from a subspace $X$ of $P$ into $P$ is called a proximity map [2], p. 124, if $f(x) \in V(x)$ for all $x \in X$. If several triangulations of $P$ are used, then we clarify our notation by writing $\tau \in(P, T)$ and by calling $f$ a $T$-proximity map. Note that if $T_{1}$ refines $T_{0}$, then a $T_{1}$-proximity map is a fortiori a $T_{0}$-proximity map.

Let $P^{I}$ be the path space of $P$ with the compact-open topology. Then a path field on $P$ is a map $\beta: P \rightarrow P^{I}$ so that $\beta(x)$ is either a path $p: I \rightarrow P$ with $p(0)=x$ and $p(s) \neq x$ for $0<s \leq 1$, or the constant path at $x$. If $\beta(x)$ is the constant path at $x$, then $x$ is called a singularity of $\beta$. A proximity map $f: P \rightarrow P$ determines a path field $\beta$ on $P$ consisting of broken line segments in such a way that the fixed points of $f$ are precisely the singularities of $\beta$. (See [9], Lemma 1.1, [2], p. 124, or [5], Lemma 2.1.) 
A homotopy $H: P \times I \rightarrow P$, or more generally its restriction $H \mid Y$ : $Y \rightarrow P$ to a subspace $Y$ of $P \times I$, is called a proximity map if $H \mid Y \cap$ $(P \times t)$ is a proximity map for every $t \in I$. A homotopy $H: P \times I \rightarrow P$ defines as usual a map $\mathbf{H}: P \times I \rightarrow P \times I$ given by $\mathbf{H}(x, t)=(H(x, t), t)$ for all $x \in P$ and $t \in I$, and hence if $H$ is a proximity map, then it determines a path field $\gamma: P \times I \rightarrow(P \times I)^{I}$ which is level-preserving in the sense that $\gamma(x, t)(s) \subset P \times t$ for all $(x, t) \in P \times I$ and $0 \leq s \leq 1$.

The following three simple lemmas concerning extensions of proximity maps will be used in the proof of the Theorem. The symbols bd and Bd denote the boundary in $P$ and in $P \times I$. Similarly we use $\mathrm{cl}, \mathrm{Cl}$, int and Int for the closure and interior in $P$ and $P \times I$.

Lemma 1. Let $\tau \in(P, T)$ have dimension at least one. Then every fixed point free proximity map $G_{0}:(\bar{\tau} \times 0) \cup(\dot{\tau} \times I) \rightarrow P$ has an extension $G$ : $\bar{\tau} \times I \rightarrow P$ which is a fixed point free proximity map.

Proof. In the construction given in the proof of Lemma 2.1 in [3] replace $f_{0}: X \rightarrow X$ by $G_{0} \mid \bar{\tau}: \bar{\tau} \rightarrow V(\tau)$ and $A$ by $\dot{\tau}$.

LEMMa 2. Let $P_{1}$ and $P_{2}$ be ( possibly empty) subpolyhedra of a compact polyhedron $(P, T)$ with $P_{2} \subset P_{1} \subset P$. Given $G_{0}:(P \times 0) \cup\left(P_{2} \times I\right) \rightarrow P$ so that $G_{0} \mid\left(\left(P_{1}-P_{2}\right) \times 0\right) \cup\left(\mathrm{bd} P_{2} \times I\right)$ is a fixed point free proximity map, then there exists an extension $G:(P \times 0) \cup\left(P_{1} \times I\right) \rightarrow P$ of $G_{0}$ so that $G \mid\left(P_{1}-P_{2}\right) \times I$ is a fixed point free proximity map.

Proof. For every vertex $v$ of $P_{1}-P_{2}$, let $G(v, t)=G_{0}(v, 0)$ for all $t \in I$. Then use Lemma 1 to extend $G_{0}$ over all $\tau \times I$, where $\tau \in P_{1}-P_{2}$ is of dimension one, then over all $\tau \times I$ where $\tau \in P_{1}-P_{2}$ is of dimension two, and continue thus until $G$ is obtained.

LEMMa 3. Let $P_{1}$ be a subpolyhedron of the compact polyhedron $(P, T)$ and $I_{1}$ a closed subinterval of $I$. Then every proximity map $G_{0}: \operatorname{Bd}\left(P_{1} \times I_{1}\right)$ $\rightarrow P$ has an extension $G: P_{1} \times I_{1} \rightarrow P$ which is a proximity map.

Proof. Let $I_{1}=\left[t_{0}, t_{1}\right]$, where $0 \leq t_{0}<t_{1} \leq 1$. As $V(v)$ is path-connected for every vertex $v \in$ int $P_{1}$, we can define $G \mid v \times I$ as any path in $V(v)$ from $G_{0}\left(v, t_{0}\right)$ to $G_{0}\left(v, t_{1}\right)$. Then we can define $G$ on all $\bar{\tau} \times I_{1}$, where $\tau \in$ int $P_{1}$ is of dimension one, as any extension of the already constructed map from $\operatorname{Bd}\left(\tau \times I_{1}\right)$ into the contractible space $V(\tau)$. Proceeding in this way over all simplexes of int $P_{1}$ of increasing dimension we obtain $G$. 
3. The proof of the theorem. We can assume that $C \neq P \times I$, as the case $C=P \times I$ is trivial. The proof of the Theorem will then be achieved in three steps. In the first we construct, for every $n=1,2,3, \ldots$, a piecewise linear arc $S_{n}$ contained in the $2^{2-n}$-neighbourhood $U\left(C, 2^{2-n}\right)$ of $C$, and in the second homotopies $F_{n}: P \times I \rightarrow P$ which are proximity maps with Fix $F_{n} \subset U\left(C, 2^{2-n}\right)$. The arc $S_{n}$ serves as a guide to ensure this condition, as in fact Fix $F_{n}$ is contained in a suitable neighbourhood of $S_{n}$. In the final step the level-preserving path fields associated with the proximity maps $F_{n}$ are used to construct homotopies $H_{n}: P \times I \rightarrow P$ with Fix $H_{n} \subset K \cup U\left(C, 2^{2-n}\right)$ and converging to a homotopy $H$ with Fix $H$ $=K$.

Step 1. Let $T_{0}$ be the given triangulation of the polyhedron $P$ and $d_{P}$ the barycentric metric of $P$ with respect to $T_{0}$. Choose refinements $T_{n}$ of $T_{n-1}$ so that mesh $\mu\left(T_{1}\right) \leq \frac{1}{2}$ and $\mu\left(T_{n}\right) \leq \frac{1}{2} \mu\left(T_{n-1}\right)$ for $n=2,3,4, \ldots$, and let $T_{n}^{\prime}$ be the triangulation of $I$ which has the vertices $v^{\prime}=l / 2^{n}$, $l=0,1, \ldots, 2^{n}$. Denote by $\mathrm{st}_{n} v$ the open star of the vertex $v \in\left(P, T_{n}\right)$, by $\mathrm{st}_{n} v^{\prime}$ the open star of $v^{\prime} \in\left(I, T_{n}^{\prime}\right)$, and define for $n=1,2, \ldots$

$$
U_{n}=\bigcup\left\{\mathrm{st}_{n} v \times \mathrm{st}_{n} v^{\prime} \mid\left(\mathrm{st}_{n} v \times \mathrm{st}_{n} v^{\prime}\right) \cap C \neq \varnothing\right\} .
$$

Then $U_{n}$ is an open and arc-connected neighbourhood of $C$. Let $U(C, \varepsilon)$ $=\{(x, t) \in P \times I \mid d((x, t), C)<\varepsilon\}$, where $d$ is the product metric of $d_{p}$ and the Euclidean metric of $I$. One can check that

$$
C \subset U_{n+1} \subset \mathrm{Cl} U_{n+1} \subset U_{n} \subset U\left(C, 2^{2-n}\right)
$$

for every $n \geq 1$. We also define $U_{0}=U_{-1}=P \times I$.

Now choose a maximal simplex $\tau_{1} \in\left(P, T_{1}\right)$ so that $C \cap\left(\bar{\tau}_{1} \times 0\right) \neq$ $\varnothing$, and for every $n \geq 2$ a maximal simplex $\tau_{n} \in\left(P, T_{n}\right)$ so that $\bar{\tau}_{n} \subseteq \bar{\tau}_{n-1}$. Similarly choose a maximal simplex $\sigma_{1} \in\left(P, T_{1}\right)$ so that $C \cap\left(\bar{\sigma}_{1} \times 1\right) \neq$ $\varnothing$, and for every $n \geq 2$ a maximal simplex $\sigma_{n} \in\left(P, T_{n}\right)$ so that $\bar{\sigma}_{n} \subset \bar{\sigma}_{n-1}$. Select, for all $n \geq 1$, points $p_{n} \in \tau_{n} \times 0$ and $q_{n} \in \sigma_{n} \times 1$. Then it is possible to construct a piecewise linear arc $S_{n} \subset U_{n}$ with the following properties (compare Figure 1, in which the non-maximal and maximal simplexes of $\left(P, T_{n}\right)$ are schematically represented by points and line segments):

(i) $S_{n} \cap(P \times 0)=p_{n}$ and $S_{n} \cap(P \times 1)=q_{n}$,

(ii) $S_{n}$ is a step-polygon consisting of subarcs joined end-to-end. Each subarc is either a vertical segment contained in $p \times I$, where $p$ is constant and contained in a maximal simplex of $\left(P, T_{n}\right)$, or it is a horizontal subarc contained in $P \times(2 l-1) / 2^{n+1}$, where $l$ (with $1 \leq l \leq 2^{n}$ ) is an integer, 
(iii) no closed simplex of $\left(P \times(2 l-1) / 2^{n+1}, T_{n}\right)$ intersects two distinct horizontal subarcs of $S_{n}$.

The existence of $S_{n}$ follows from the arc-connectedness of $U_{n}$ and a general position argument. We orient each $\operatorname{arc} S_{n}$ so that its initial point is $p_{n}$ and its endpoint is $q_{n}$.

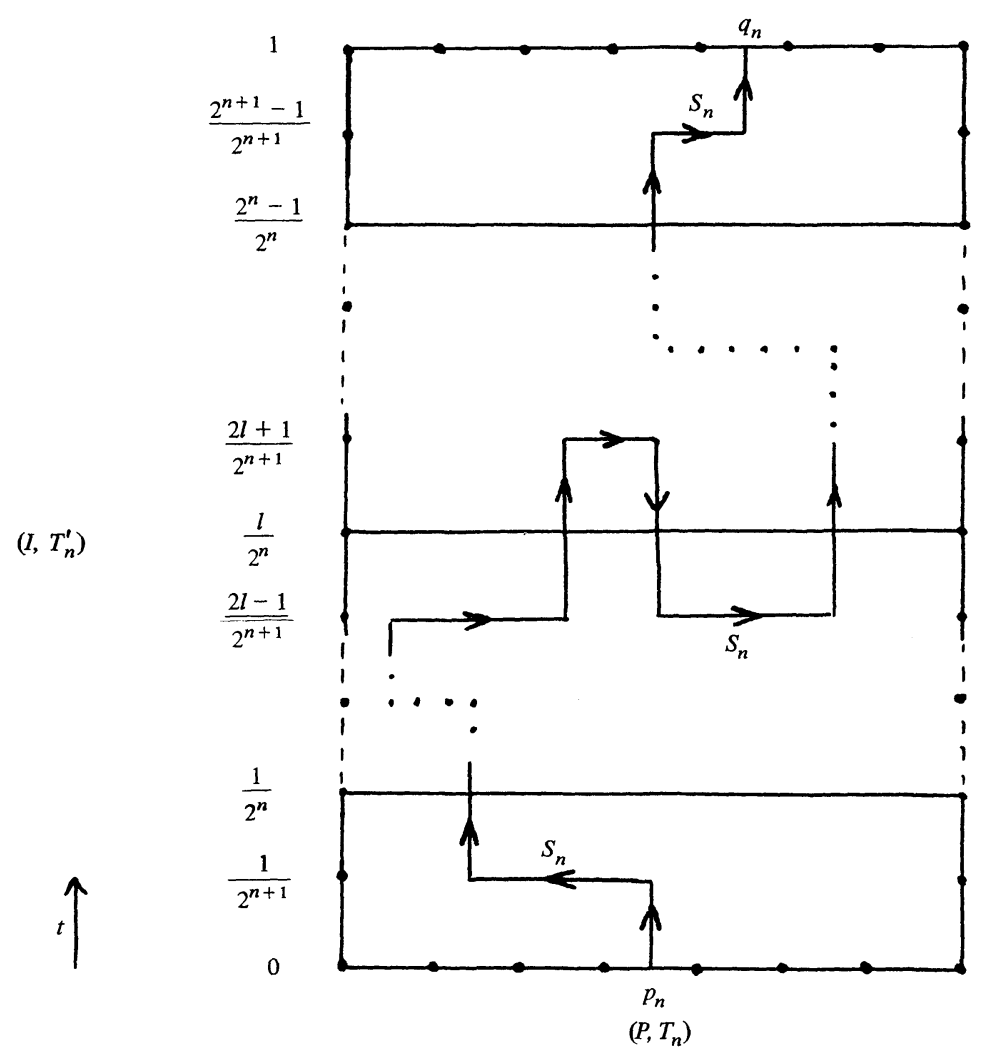

FIGURE 1. The oriented $\operatorname{arc} S_{n}$.

Step 2. The aim of this step is to construct for all $n \geq 0$ homotopies $F_{n}: P \times I \rightarrow P$ which satisfy the following conditions if $n \geq 1$ :

$\left(F_{n}, 1\right)$ Fix $F_{n} \subset U_{n}$,

$\left(F_{n}, 2\right) F_{n} \mid \mathrm{Cl} U_{n-1}$ is a $T_{n}$-proximity map,

$\left(F_{n}, 3\right) F_{n} \mid \mathrm{Cl} U_{n-2}$ is a $T_{n-1}$-proximity map,

$\left(F_{n}, 4\right) F_{n}=F_{n-1}$ on $P \times I-U_{n-2}$.

Using the fact that $U_{n+1} \subset U_{n}$ for all $n \geq 0$ and the assumption $C \neq P \times I$, we can determine an integer $N \geq 0$ so that

$$
U_{0}=U_{1}=\cdots=U_{N}=P \times I \text { but } U_{N+1} \neq P \times I .
$$


According to [10], Satz 1, p. 568 (see also the proof of Ch. VIII E, Theorem 1 in [2] or of Theorem 1.4 in [9]) there exists a $T_{N+1}$-proximity map $f: P \rightarrow P$ with Fix $f=\left\{p_{N}\right\}$. We define $F_{n}$ for $n \leq N$ by $F_{n}(x, t)=$ $f(x)$ for all $(x, t) \in P \times I$. Then we proceed by induction. To derive $F_{n+1}$ from $F_{n}$ for $n \geq N$ we first change the homotopy $F_{n}$, which satisfies conditions $\left(F_{n}, 1\right)$ to $\left(F_{n}, 4\right)$ if $n \geq 1$, to a homotopy $G_{n}: P \times I \rightarrow P$ which satisfies the following conditions:

$\left(G_{n}, 1\right)$ Fix $G_{n}=$ Fix $F_{n}$,

$\left(G_{n}, 2\right) G_{n} \mid \mathrm{Cl} U_{n}$ is a $T_{n+1}$-proximity map,

$\left(G_{n}, 3\right) G_{n} \mid \mathrm{Cl} U_{n-1}$ is a $T_{n}$-proximity map,

$\left(G_{n}, 4\right) G_{n}=F_{n}$ on $P \times I-U_{n-1}$.

To do so, choose for all $n \geq N$ an $\varepsilon_{n}>0$ so that a homotopy $G$ : $P \times I \rightarrow P$ with $d_{P}(x, G(x, t))<\varepsilon_{n}$ for all $(x, t) \in P \times I$ is a $T_{n}$-proximity map. The definition of $F_{n}$ for $n \leq N$ and the inductive conditions $\left(F_{n}, 2\right)$ to $\left(F_{n}, 4\right)$ imply that each $F_{n}: P \times I \rightarrow P$ is a $T_{1}$-proximity map and thus determines a level-preserving path field $\gamma_{n}: P \times I \rightarrow(P \times I)^{I}$. Let $\pi$ : $P \times I \rightarrow P$ be the projection and select $s_{n}$ with $0<s_{n}<1$ so that $d_{P}\left(x, \pi \circ \gamma_{n}(x, t)(s)\right)<\varepsilon_{n+1}$ for all $s \leq s_{n}$ and $(x, t) \in P \times I$. Then define $G_{n}$ for $n=N, N+1$ and all $(x, t) \in P \times I$ by

$$
\begin{aligned}
G_{N}(x, t) & =F_{N}(x, t), \\
G_{N+1}(x, t) & =\pi \circ \gamma_{N+1}(x, t)\left(s_{N+1}\right) .
\end{aligned}
$$

For $n \geq N+2$ we have $\mathrm{Cl} U_{n} \subset U_{n-1} \neq P \times I$, hence there exists a map $w_{n}: P \times I \rightarrow\left[s_{n}, 1\right]$ so that $\mathrm{Cl} U_{n} \subset w_{n}^{-1}\left(s_{n}\right)$ and $P \times I-U_{n-1} \subset w_{n}^{-1}(1)$. If we define $G_{n}$ for $n \geq N+2$ by

$$
G_{n}(x, t)= \begin{cases}F_{n}(x, t) & \text { if }(x, t) \in P \times I-U_{n-1}, \\ \pi \circ \gamma_{n}(x, t)\left(w_{n}(x, t)\right) & \text { if }(x, t) \in \mathrm{Cl} U_{n-1},\end{cases}
$$

then $G_{n}$ is defined for all $n \geq N$ as a map which satisfies $\left(G_{n}, 1\right)$ to $\left(G_{n}, 4\right)$.

To construct $F_{n+1}$ from $G_{n}$ for $n \geq N$, we first use [2], Ch. VIII C, Lemma 2 to obtain $F_{n+1} \mid P \times 0$ from $G_{n} \mid P \times 0$ by moving the fixed point $p_{n}$ within its carrier $\kappa_{n}$ in $\left(P \times 0, T_{n}\right)$ to $p_{n+1}$ in such a way that $F_{n+1} \mid \bar{\kappa}_{n} \times 0$ is still a $T_{n+1}$-proximity map and that $F_{n+1} \mid\left(P-\kappa_{n}\right) \times 0=$ $G_{n} \mid\left(P-\kappa_{n}\right) \times 0$. Then we construct $F_{n+1} \mid P \times\left[0,(l+1) / 2^{n+1}\right]$ from $F_{n+1} \mid P \times\left[0, l / 2^{n+1}\right]$ by induction on $l=0,1, \ldots, 2^{n+1}-1$. First we extend $F_{n+1}$ over $\left(P \times I-U_{n}\right) \cap\left(P \times I_{n+1, l}\right)$, where

$$
I_{n+1, l}=\left[l / 2^{n+1},(l+1) / 2^{n+1}\right] \text {, }
$$

by

$$
F_{n+1}(x, t)=G_{n}(x, t) \text { for all }(x, t) \in\left(P \times I-U_{n}\right) \cap\left(P \times I_{n+1, l}\right) .
$$


The oriented arc $S_{n+1}$ is now used to define $F_{n+1}$ on $\mathrm{Cl} U_{n} \cap\left(P \times I_{n+1, l}\right)$ so that Fix $F_{n+1} \subset U_{n+1}$. To ensure that Fix $F_{n+1}$ is a set near $S_{n+1} \subset U_{n+1}$, we shall in the induction on $l$ assume that $F_{n+1}$ satisfies not only $\left(F_{n+1}, 1\right)$ to $\left(F_{n+1}, 4\right)$, but also the further condition $(F, l)$, in which $\chi(P)$ denotes the Euler characteristic of $P$. (Compare with Figure 2, in which $S^{\prime}$ represents a component of $S_{n+1} \cap\left(P \times I_{n+1, l}\right)$.)

$(F, l)$ Fix $F_{n+1} \cap\left(P \times l / 2^{n+1}\right)=S_{n+1} \cap\left(P \times l / 2^{n+1}\right)$, and each of those finitely many fixed points have index $\chi(P)$ (index $-\chi(P)$ ) if the $t$-coordinate of $S_{n+1}$ is increasing (decreasing) at this point.

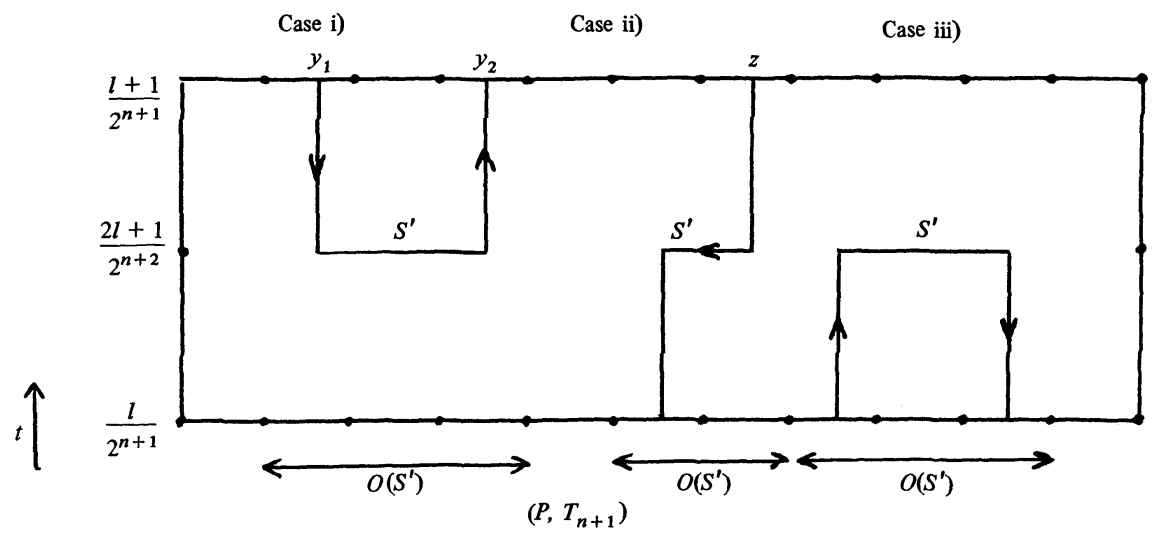

Figure 2. A part of $P \times I_{n+1, l}$.

Clearly $(F, l)$ holds for $l=0$. We now proceed with the construction of $F_{n+1}$ on $\mathrm{Cl} U_{n} \cap\left(P \times I_{n+1, l}\right)$. The set $S_{n+1} \cap\left(P \times I_{n+1, l}\right)$ consists of one or more components which are subarcs of $S_{n+1}$. Let $S^{\prime}=S_{n+1}^{\prime}$ be one of them, let $\mathrm{st}_{n+1} \tau$ be the open star of the simplex $\tau \in\left(P, T_{n+1}\right)$, and define an open subset $O\left(S^{\prime}\right)=O_{n+1, l}\left(S^{\prime}\right)$ of $P$ by

$$
O\left(S^{\prime}\right)=\bigcup\left\{\mathrm{st}_{n+1} \tau \mid\left(\tau \times \frac{2 l+1}{2^{n+2}}\right) \cap S^{\prime} \neq \varnothing\right\} .
$$

Then $S_{n+1} \subset U_{n+1}$ implies that $O\left(S^{\prime}\right) \times\left(l / 2^{n+1}, l+1 / 2^{n+1}\right) \subset U_{n+1}$, and $(F, l)$ implies that $F_{n+1}$ is fixed point free on bd $O\left(S^{\prime}\right) \times l / 2^{n+1}$. Hence we can use Lemma 2 , with $P_{2}=\varnothing$ and

$$
P_{1}=\bigcup\left\{\text { bd } O\left(S^{\prime}\right) \mid S^{\prime} \text { is a component of } S_{n+1} \cap\left(P \times I_{n+1, l}\right)\right\}
$$

to extend $F_{n+1}$ as a fixed point free $T_{n+1}$-proximity map over $P_{1} \times I_{n+1, l}$. Now select one component $S^{\prime}$ of $S_{n+1} \cap\left(P \times I_{n+1, l}\right)$. As $P$ has no local cut points, $O\left(S^{\prime}\right)$ is 2-dimensionally connected. Note also that condition (iii) in the construction of $S_{n}$ implies that $O\left(S_{1}^{\prime}\right) \cap O\left(S_{2}^{\prime}\right)=\varnothing$ if $S_{1}^{\prime}, S_{2}^{\prime}$ are two distinct components of $S_{n+1} \cap\left(P \times I_{n+1, l}\right)$. Therefore 
$F_{n+1} \mid$ bd $O\left(S^{\prime}\right) \times(l+1) / 2^{n+1}$ has an extension to $F_{n+1}^{\prime}: \operatorname{cl} O\left(S^{\prime}\right) \times$ $(l+1) / 2^{n+1} \rightarrow P$ which is a $T_{n+1}$-proximity map with only one fixed point which lies in a maximal simplex of $\left(P \times(l+1) / 2^{n+1}, T_{n+1}\right)$. (See [10], Satz 1, or the proof of Theorem 1 in Ch. VIII E of [2].) The change of $F_{n+1}^{\prime}$ to a map $F_{n+1}$ satisfying $(F, l+1)$ depends on the cardinality of $S_{n+1} \cap\left(O\left(S^{\prime}\right) \times(l+1) / 2^{n+1}\right)$ (compare Figure 2$)$.

(i) $S_{n+1} \cap\left(O\left(S^{\prime}\right) \times(l+1) / 2^{n+1}\right)$ consists of two points. Then $S_{n+1}$ enters $O\left(S^{\prime}\right) \times I_{n+1, l}$ through one of them (say $y_{1}$ ) and exits through the other (say $\left.y_{2}\right)$. Hence $S_{n+1} \cap\left(\operatorname{cl} O\left(S^{\prime}\right) \times l / 2^{n+1}\right)=\varnothing$, and so $(F, l)$ implies that

$$
\operatorname{ind}\left(F_{n+1}^{\prime}, \operatorname{cl} O\left(S^{\prime}\right) \times l / 2^{n+1}\right)=\operatorname{ind}\left(F_{n+1}, \operatorname{cl} O\left(S^{\prime}\right) \times l / 2^{n+1}\right)=0 .
$$

As

$$
F_{n+1}^{\prime}\left|\operatorname{bd} O\left(S^{\prime}\right) \times I_{n+1, l}=F_{n+1}\right| \text { bd } O\left(S^{\prime}\right) \times I_{n+1, l}
$$

is fixed point free, it follows from the homotopy property of the index that ind $\left(F_{n+1}^{\prime}, \operatorname{cl} O\left(S^{\prime}\right) \times(l+1) / 2^{n+1}\right)=0$. So we can use the proof of Lemma 1 in [6] to split the fixed point of $F_{n+1}^{\prime}$ into two points, one of them of index $\chi(P)$ and the other of index $-\chi(P)$, and then use [2], Ch. VIII C, Lemmas 2 and 3, to move the fixed point of index $-\chi(P)$ to $y_{1}$ and the one of index $\chi(P)$ to $y_{2}$. Thus we change $F_{n+1}^{\prime}$ to a map $F_{n+1} \mid \operatorname{cl} O\left(S^{\prime}\right) \times(l+1) / 2^{n+1}$ which satisfies $\left(F_{n+1}, 1\right)$ to $\left(F_{n+1}, 4\right)$ and $(F, l+1)$.

(ii) $S_{n+1} \cap\left(O\left(S^{\prime}\right) \times(l+1) / 2^{n+1}\right)$ consists of one point $z$. In this case we change $F_{n+1}^{\prime}$ to $F_{n+1} \mid \operatorname{cl} O\left(S^{\prime}\right) \times(l+1) / 2^{n+1}$ by moving the fixed point of $F_{n+1}^{\prime}$ to $z$. An argument similar to the one in the first case shows that it will have the index required by $(F, l+1)$.

(iii) $S_{n+1} \cap\left(O\left(S^{\prime}\right) \times(l+1) / 2^{n+1}\right)=\varnothing$. This means that $S_{n+1}$ both enters and exits from $O\left(S^{\prime}\right) \times I_{n+1, l}$ through $O\left(S^{\prime}\right) \times l / 2^{n+1}$, hence $\operatorname{ind}\left(F_{n+1}^{\prime}, \operatorname{cl} O\left(S^{\prime}\right) \times(l+1) / 2^{n+1}\right)=\operatorname{ind}\left(F_{n+1}, \operatorname{cl} O\left(S^{\prime}\right) \times l / 2^{n+1}\right)=0$.

Therefore we can use [2], Ch. VIII B, Theorem 4 to eliminate the fixed point of $F_{n+1}^{\prime}$ and thus obtain $F_{n+1} \mid \mathrm{cl} O\left(S^{\prime}\right) \times(l+1) / 2^{n+1}$ as desired.

$F_{n+1}$ is now defined on $\operatorname{Bd}\left(O\left(S^{\prime}\right) \times I_{n+1, l}\right)$ as a $T_{n+1}$-proximity map. We choose $F_{n+1} \mid \mathrm{cl} O\left(S^{\prime}\right) \times I_{n+1, l}$ as any extension which is a $T_{n+1}$-proximity map; it exists according to Lemma 3.

Proceeding thus we can define $F_{n+1}$ on

$$
\begin{array}{r}
R_{n+1, l}=\bigcup\left\{\mathrm{cl} O\left(S^{\prime}\right) \times I_{n+1, l} \mid S^{\prime}\right. \text { is a component of } \\
\left.S_{n+1} \cap\left(P \times I_{n+1, l}\right)\right\} .
\end{array}
$$


As $R_{n+1, l} \subset \mathrm{Cl} U_{n+1}$, we can finally use Lemma 2, with $P=P_{1}$ and $R_{n+1, l} \cup\left[\left(P \times I-U_{n}\right) \cap\left(P \times I_{n+1, l}\right)\right]$ replacing $P_{2} \times I$ to obtain $F_{n+1} \mid P \times I_{n+1, l}$ and hence $F_{n+1} \mid\left[0,(l+1) / 2^{n+1}\right]$. By induction on $l$ we construct a homotopy $F_{n+1}: P \times I \rightarrow P$ which satisfies $\left(F_{n+1}, 1\right)$ to $\left(F_{n+1}, 4\right)$.

Step 3. We shall construct a homotopy $H: P \times I \rightarrow P$ with given fixed point set $K$, where $C \subset K \subset P \times I$, as the limit of homotopies $H_{n}$ : $P \times I \rightarrow P$ with Fix $H_{n} \subset K \cup U_{n}$. To obtain $H_{n}$ from $F_{n}$, we use the fact that each $F_{n}: P \times I \rightarrow P$ is a $T_{1}$-proximity map and hence determines a level-preserving path field $\gamma_{n}: P \times I \rightarrow(P \times I)^{I}$.

We change the product metric $d$ (which was defined in Step 1) to the bounded metric $\bar{d}=\min (1, d)$, and parametrize all $\gamma_{n}(x, t)$ by $s \in I$ so that

$$
\rho\left(\gamma_{n}(x, t)(0), \gamma_{n}(x, t)(s)\right)=s \rho\left(\gamma_{n}(x, t)(0), \gamma_{n}(x, t)(1)\right),
$$

where $\rho$ is the arc length with respect to the metric $d_{p}$ measured along the piecewise linear path $\gamma_{n}(x, t)$. Let homotopies $H_{n}: P \times I \rightarrow P$ be defined by

$$
H_{n}(x, t)=\gamma_{n}(x, t)(\bar{d}((x, t), K)) \quad \text { for all }(x, t) \in P \times I \text { and } n \geq 0 .
$$

As the singularities of $\gamma_{n}$ are at Fix $F_{n} \subset U_{n}$, we have Fix $H_{n} \subset K \cup U_{n}$. To see that the $H_{n}$ converge, note that it follows from $\left(F_{n}, 4\right)$ that

$$
H_{n+1}=H_{n} \quad \text { on } P \times I-U_{n-1} \text {. }
$$

If $(x, t) \in U_{n-1} \subset U\left(C, 2^{3-n}\right)$, then $d((x, t), K) \leq d((x, t), C) \leq 2^{3-n}$. So if $n \geq 3$, then $\bar{d}((x, t), K)=d((x, t), K)$ and therefore

$$
\begin{aligned}
d_{P}\left(H_{n+1}(x, t), H_{n}(x, t)\right) & \leq d_{P}\left(H_{n+1}(x, t), x\right)+d_{P}\left(H_{n}(x, t), x\right) \\
& \leq 2 \cdot 2 \mu\left(T_{1}\right) \cdot 2^{3-n} \leq 2^{4-n} .
\end{aligned}
$$

Thus we have for all $n \geq 3$

$$
\sup \left\{d_{P}\left(H_{n+1}(x, t), H_{n}(x, t)\right) \mid(x, t) \in P \times I\right\} \leq 2^{4-n},
$$

and $\left\{H_{n}\right\}$ is a Cauchy sequence in the sup metric. This allows us to define a homotopy $H=\lim _{n \rightarrow \infty} H_{n}$. It is easy to check that Fix $H_{n} \subset K \cup U_{n} \subset$ $K \cup U\left(C, 2^{3-n}\right)$ implies that Fix $H=K$, so $H$ is a homotopy as required in the Theorem.

4. Remarks. We want to point out that the proof given here, which is based on a technique developed in connection with problems concerning the CIP, does not allow one to impose any conditions on the 
homotopy. We have obtained $H$ so that $H \mid P \times t$ is, for every $t \in I$, homotopic to the identity map of $P$. Hence many questions concerning fixed point sets of homotopies remain unanswered. Here are some.

(i) What conditions does $K$ have to satisfy so that it can be realized as the fixed point set of a homotopy $H: f \simeq g$ if one or both of $f$ and $g$ are prescribed? Even if the given maps $f$ and $g$ are homotopic to the identity the method of our proof does not provide an answer.

(ii) What conditions does $K$ have to satisfy so that it can be realized as the fixed point set of a homotopy $H: f \simeq g$ if the homotopy class [ $f]$ of $f$ is prescribed and differs from the identity class? It is known that then the closed set $K$ must contain at least $N(f)$ continua joining $P \times 0$ and $P \times 1$ (this fact can either be obtained as a consequence of recent work by E. Fadell or as a fairly easy extension of [1], Theorem 1), but it is also known that this condition is not sufficient. As even the corresponding simpler problem for maps-i.e. the problem of realizing a closed set of a selfmap $f$ of $P$ with prescribed homotopy class [ $f]$-has not yet been satisfactorily solved, this question may be premature.

(iii) If $P$ admits a fixed point free map $f$, then obviously $H(x, t)=f(x)$ for all $t \in I$ defines a homotopy $H$ with Fix $H=\varnothing$. But is it always possible to obtain a fixed point free homotopy $H: f \simeq g$ if $f$ and $g$ are prescribed fixed point free maps? A answer to this question is not even known if $f$ and $g$ are small deformations of the identity.

(iv) Question (iii) concerns a special case of the problem of minimizing the fixed point set of a homotopy $H: f \simeq g$ if $f$ and $g$ have minimal fixed point sets. A more general question is the following: If $f$ and $g$ are two homotopic maps so that each has $N(f)$ (= Nielsen number of $f$ ) fixed points, does there exist a homotopy $H: f \simeq g$ so that $H \mid P \times t$ has only $N(f)$ fixed points for all $t \in I$ ? It is only known that $H$ can be chosen so that $H \mid P \times t$ has a finite fixed point set for all $t \in I$ [7], which is a much weaker result.

\section{REFERENCES}

1. F. E. Browder, On continuity of fixed points under deformations of continuous mappings, Summa Brasil. Math., 4 (1960), 183-191.

2. R. F. Brown, The Lefschetz Fixed Point Theorem, Scott, Foresman and Co., Glenview, I11., 1971.

3. Boju Jiang (Po-chu Chiang), On the least number of fixed points, Amer. J. Math., 102 (1980), 749-763.

4. Boju Jiang and $\mathrm{H}$. Schirmer, Fixed point sets of continuous selfmaps of polyhedra, Fixed Point Theory, Proceedings, Sherbrooke, Québec, 1980, Lecture Notes in Math., v. 886, Springer-Verlag, Berlin, Heidelberg, New York.

5. E. Fadell, A remark on simple path fields in polyhedra of characteristic zero, Rocky Mountain J. Math., 4 (1974), 65-68. 
6. H. Schirmer, Mappings of polyhedra with prescribed fixed points and fixed point indices, Pacific J. Math., 63 (1976), 521-530.

7. __ Fix-finite homotopies, Pacific J. Math., 83 (1979), 531-542.

8. __ Fixed point sets of continuous selfmaps, Fixed Point Theory, Proceedings, Sherbrooke, Québec, 1980, Lecture Notes in Math., v. 886, Springer-Verlag, Berlin, Heidelberg, New York.

9. Shi Gen-Hua, On least number of fixed points and Nielsen numbers, Acta Math. Sinica, 16 (1966), 223-232 (= Chinese Math.-Acta, 8 (1966), 234-243).

10. F. Wecken, Fixpunktklassen III, Math. Ann., 118 (1942), 544-577.

Received October 30, 1981 and in revised form March 31, 1982. This research was partially supported by NSERC Grant A 7579.

CARLETON UNIVERSITY

OtTawa, Canada 


\section{PACIFIC JOURNAL OF MATHEMATICS EDITORS}

Donald BABBITT (Managing Editor)

University of California

Los Angeles, CA 90024

Hugo Rossi

University of Utah

Salt Lake City, UT 84112

C. C. Moore and Arthur Ogus

University of California

Berkeley, CA 94720
J. DugundiI

Department of Mathematics

University of Southern California

Los Angeles, CA 90089-1113

R. Finn and H. SAMELSON

Stanford University

Stanford, CA 94305

ASSOCIATE EDITORS
R. ARENS
E. F. BECKENBACH
B. H. NeUmanN
F. WOLF
K. YosHIDA (1906-1982)

\section{SUPPORTING INSTITUTIONS}

UNIVERSITY OF ARIZONA

UNIVERSITY OF BRITISH COLUMBIA

CALIFORNIA INSTITUTE OF TECHNOLOGY

UNIVERSITY OF CALIFORNIA

MONTANA STATE UNIVERSITY

UNIVERSITY OF NEVADA, RENO

NEW MEXICO STATE UNIVERSITY

OREGON STATE UNIVERSITY
UNIVERSITY OF OREGON

UNIVERSITY OF SOUTHERN CALIFORNIA

STANFORD UNIVERSITY

UNIVERSITY OF HAWAII

UNIVERSITY OF TOKYO

UNIVERSITY OF UTAH

WASHINGTON STATE UNIVERSITY

UNIVERSITY OF WASHINGTON 


\section{Pacific Journal of Mathematics}

\section{Vol. 108, No. $1 \quad$ March, 1983}

Waleed A. Al-Salam and A. Verma, $q$-Konhauser polynomials $\ldots \ldots \ldots \ldots 1$

Alfred David Andrew, The Banach space JT is primary $\ldots \ldots \ldots \ldots \ldots . . .6$

Thomas E. Bengtson, Bessel functions on $P_{n} \ldots \ldots \ldots \ldots \ldots \ldots$

Joaquim Bruna Floris and Francesc Tugores, Free interpolation for

holomorphic functions regular to the boundary $\ldots \ldots \ldots \ldots \ldots \ldots \ldots \ldots$

Peter Dierolf and Susanne Dierolf, Topological properties of the dual pair

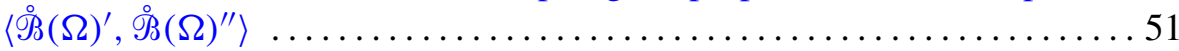

Gerald Arthur Edgar, An ordering for the Banach spaces $\ldots \ldots \ldots \ldots \ldots 83$

Basil Gordon, A proof of the Bender-Knuth conjecture . . . . . . . . . . . . . 99

Harold T. Hodes, A minimal upper bound on a sequence of Turing degrees

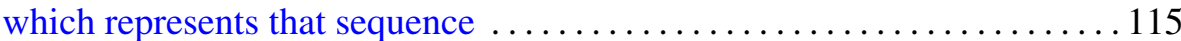

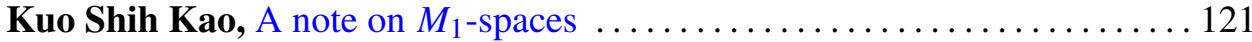

Frank Kost, Topological extensions of product spaces ................ 129

Eva Lowen-Colebunders, On the convergence of closed and compact

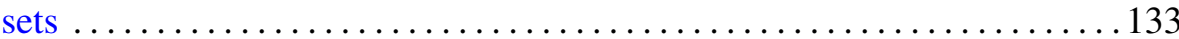

Doron Lubinsky, Divergence of complex rational approximations . . . . . . 141

Warren May and Elias Hanna Toubassi, Endomorphisms of rank one

mixed modules over discrete valuation rings $\ldots \ldots \ldots \ldots \ldots \ldots \ldots \ldots \ldots$

Richard Patrick Morton, The quadratic number fields with cyclic

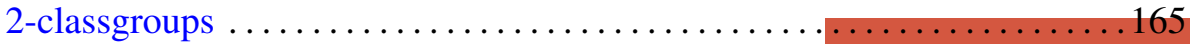

Roderic Murufas, Rank of positive matrix measures . . . . . . . . . . . . 177

Helga Schirmer, Fixed point sets of homotopies . . . . . . . . . . . . . 191

E. Taflin, Analytic linearization of the Korteweg-de Vries equation ........ 203

James Thomas Vance, Jr., $L^{p}$-boundedness of the multiple Hilbert

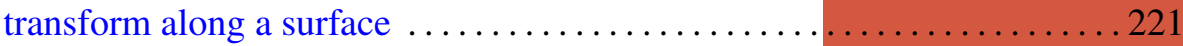

Hiroshi Yamaguchi, A property of some Fourier-Stieltjes transforms . . . . . 243 